\title{
Large Eddy Simulation of Three- Dimensional Turbulent Flows by the Finite Element Method
}

Adriane P. Petry

adrianep@mecanica.ufrgs.br Departamento de Engenharia Mecânica Universidade Federal do Rio Grande do Sul 90050-170 Porto Alegre, RS, Brazil

\section{Armando M. Awruch \\ Sênior Member, ABCM \\ Programa de Engenharia Civil Universidade Federal do Rio Grande do Sul 90035-190 Porto Alegre, RS, Brazil}

The main objectives of this work are the formulation, implementation and applications of a numerical algorithm to simulate turbulent, incompressible, isothermal flows. The transient three-dimensional flow is analyzed using an explicit Taylor-Galerkin scheme and the finite element method with hexahedrical eight-node element. Turbulence is simulated using Large Eddy Simulation. For sub-grid scales two models were implemented, the classical Smagorinsky's model and the dynamic eddy viscosity model. For the second filtration, which is necessary in the dynamic model, a new method was developed based on independent finite elements that involve each node in the original mesh. The implemented scheme is efficient and good results with low additional computational cost were obtained. Results for two classical problems, the driven cavity and the backward facing step are presented. Comments about the model applicability for flows with high Reynolds numbers are also presented.

Keywords: Turbulence, large Eddy simulation, dynamic model, finite element method, computational fluid dynamics

\section{Introduction}

Flow analysis is an important subject for several engineering fields, as well as in other areas of science and technology. Many problems are characterized by turbulent flows and suitable models are necessary to represent the flow characteristics.

High Reynolds numbers, a coherent behavior at large scales of movement and a random behavior at small scales usually characterize turbulent flows. They are also diffusive, threedimensional and transient (Tenekes and Lumley, 1972). Another important characteristic of turbulent flows is that multiple scales are involved (Silveira Neto, 2000), however even small scales are usually larger than the scales of molecular movement (Hinze, 1975), consequently turbulence may be described as a continuous phenomenon.

Using the conservation equations of mass, energy and momentum, a complex system of partial differential equations is obtained. Computational Fluid Dynamic is an important methodology to simulate complex flows governed by such systems. Different numerical methods are used in these simulations. The Finite Element Method is an efficient technique for the analysis of problems with complex geometry (Hughes, 1987; Reddy and Gartling, 1994). This methodology was adopted in the present work.

The conservation equations of fluid mechanics form a mathematical model that is capable of describe turbulent fluid flows. However, the required discretization in space and time to simulate all scales directly (Direct Simulation) does not permit the application of this procedure for most practical problems. This is a consequence of the large number of equations to be solved, leading to excessive processing time, even for the most advanced computers (Kim and Menon, 1999). As a consequence of the impossibility to apply Direct Simulation for a large range of problems, it is necessary to use alternative methodologies, such as the classic models based on the solution of the Reynolds Average Equations (Hinze, 1975) and the Large Eddy Simulation (Ferziger 1993, Rogallo and Moin 1984, Lesieur et al, 1995).

In the Large Eddy Simulation technique, the conservation equations are solved for large flow scales and models are used to represent the effects of the subgrid scales. These models have the

Presented at ETT $2004-4^{\text {th }}$ Spring School on Transition and Turbulence September $27^{\text {th }}$ - October $1^{\text {st }}, 2004$, Porto Alegre, RS, Brazil.

Paper accepted: May, 2005. Technical Editor: Aristeu da Silveira Neto. same purpose of the conventional turbulence models, but they may be simple because they consider only the effect of small scales. Moreover, subgrid models have minor geometry dependence because the small scales are of more universal nature than the total turbulence.

To overcome difficulties in the numerical analysis of incompressible flows it is necessary to adopt some scheme to stabilize the solution (Reddy and Gartling, 1994, Franca and Frey, 1992). The adopted alternative in present work is the quasiincompressible formulation (Kawahara and Hirano, 1983). The usual formulation for incompressible flow is based on the assumption of a constant value for the density and, from this hypothesis, it is derived that the speed of sound in the flow field is infinite (Schlichting, 1968). However, in real flows the propagation of sound always occurs with a finite speed. The equations for a quasi-incompressible flow assume constant density (density fluctuations are considered negligible) and a finite value for the speed of sound. With this consideration the mass conservation equation contains a time derivative of pressure.

This work presents the formulation, implementation and application of a numerical algorithm for three-dimensional, turbulent flow analysis. The methodology is based on the Finite Element Method and Large Eddy Simulation. A computational code to simulate transient, quasi-incompressible, three-dimensional flows, was developed (Petry, 2002) using an explicit TaylorGalerkin scheme, with eight-node hexahedrical element. Two different subgrid models were implemented: the Smagorinsky's model (Smagorinsky, 1963) and the dynamic model (Germano et al., 1991; Lilly, 1992). The dynamic model implementation implies in a second filter operation. A new methodology was developed for this process, called Second Filter by Independent Finite Elements. Simulations of the backward-facing step and the driven cavity flows are presented in this work. These simulations confirm the validity of the implemented scheme, however they also demonstrate that improvements are necessary to overcome difficulties for large computational problems.

\section{Nomenclature}

$$
\begin{aligned}
& C=\text { sound propagation speed, }(\mathrm{m} / \mathrm{s}) \\
& C_{S}=\text { Smagorinsky's constant } \\
& \mathrm{h}, \mathrm{H}, \mathrm{B}, \mathrm{D}, \mathrm{w}=\text { length dimensions of problems domains, }(\mathrm{m}) \\
& n_{j}=\text { cosine of the angle between the normal vector to the } \\
& \quad \text { boundary with axis } x_{j}
\end{aligned}
$$


$\overline{\mathrm{p}}=$ large scale pressure component, $(\mathrm{Pa})$

Re $=$ Reynolds number, dimensionless

$t_{i}=$ prescribed values of surface forces at the boundary

UV1, UV2 = dimensionless Reynolds stresses

$\overline{\mathrm{v}_{\mathrm{i}}}=$ large scale velocity component, $(\mathrm{m} / \mathrm{s})$

$\mathrm{v}_{\mathrm{i}}^{\prime}=$ subgrid scale velocity component, $x_{i}(\mathrm{~m} / \mathrm{s})$

$\hat{\mathrm{v}}_{\mathrm{i}}=$ prescribed values of velocity component at the boundary, $(\mathrm{m} / \mathrm{s})$

$\mathrm{V} 1$ = dimensionless mean velocity

V1rms, V2rms = dimensionless forms of the root mean square

$\mathrm{V} 2$ = dimensionless mean velocity

$\mathrm{V}_{\max }=$ maximum value of the velocity profile, $(\mathrm{m} / \mathrm{s})$

$x_{i}=$ coordinates, $i=1,2,3,(m)$

$\mathrm{Xr}=$ reattachment length, $m$

\section{Greek Symbols}

$\delta_{i j}=$ Kroenecker delta

$\lambda=$ volumetric viscosity, $\mathrm{kg} /(\mathrm{ms})$

$\mu=$ dynamic viscosity, $\mathrm{kg} /(\mathrm{m} \mathrm{s})$

$v=$ kinematic viscosity, $\mathrm{m}^{2} / \mathrm{s}$

$\rho=$ density, $\mathrm{kg} / \mathrm{m}^{3}$

$\psi_{\alpha}=$ interpolation function of node $\alpha$

$(\xi, \eta, \zeta)=$ natural coordinates

$\Omega=$ volume domain

$\Gamma_{\mathrm{v}}=$ boundary face with prescribed velocity

$\Gamma \mathrm{t}=$ face with natural boundary condition

\section{Mathematical and Numerical Aspects}

\section{Governing Equations}

From the equations of conservation of mass, energy and momentum for three-dimensional, transient, isothermal, quasiincompressible viscous flows of a Newtonian fluid (White, 1974, Kawahara and Hirano, 1983), the equations for Large Eddy Simulation are derived (Petry, 2002).

In a Large Eddy Simulations (Findikakis and Street, 1982) each field variable is decomposed into a large-scale field (identified by the over-bar) and a subgrid scale field (identified by the apostrophe):

$$
\mathrm{v}_{\mathrm{i}}=\overline{\mathrm{v}_{\mathrm{i}}}+\mathrm{v}_{\mathrm{i}}^{\prime} \quad \mathrm{p}=\overline{\mathrm{p}}+\mathrm{p}^{\prime} \quad \rho=\bar{\rho}+\rho^{\prime}
$$

Since density is constant, $\rho^{\prime}=0$.

After the filtering process the governing equations are given by:

$$
\begin{gathered}
\frac{\partial \bar{p}}{\partial t}+C^{2} \frac{\partial}{\partial x_{j}}\left(\rho \bar{v}_{j}\right)=0 \\
\frac{\partial}{\partial t}\left(\rho \bar{v}_{i}\right)+\frac{\partial}{\partial x_{j}}\left(\rho \bar{v}_{i} \bar{v}_{j}\right)+\frac{\partial \bar{p}}{\partial x_{j}} \delta_{i j}- \\
\frac{\partial}{\partial x_{j}}\left\{v\left(\frac{\partial}{\partial x_{j}}\left(\rho \bar{v}_{i}\right)+\frac{\partial}{\partial x_{i}}\left(\rho \bar{v}_{j}\right)\right)+\frac{\lambda}{\rho}\left(\frac{\partial}{\partial x_{k}}\left(\rho \bar{v}_{k}\right)\right) \delta_{i j}\right\} \\
+\frac{\partial}{\partial x_{j}}\left\{\rho\left(L_{i j}+C_{i j}+\bar{v}_{i}^{\prime} v_{j}^{\prime}\right)\right\}-f_{i}=0 \\
(i, j, k=1,2,3) \text { in } \Omega
\end{gathered}
$$

$$
\begin{gathered}
\mathrm{v}_{\mathrm{i}}=\hat{\mathrm{v}}_{\mathrm{i}} \quad(\mathrm{i}, \mathrm{j}, \mathrm{k}=1,2,3) \text { in } \Gamma \mathrm{v} \\
\left\{\begin{array}{l}
{\left[-\overline{\mathrm{p}}+\frac{\lambda}{\rho} \frac{\partial}{\partial \mathrm{x}_{\mathrm{k}}}\left(\rho \overline{\mathrm{v}_{\mathrm{k}}}\right)\right] \delta_{\mathrm{ij}}} \\
+\mathrm{v}\left[\frac{\partial}{\partial \mathrm{x}_{\mathrm{j}}}\left(\rho \overline{\mathrm{v}_{\mathrm{i}}}\right)+\frac{\partial}{\partial \mathrm{x}_{\mathrm{i}}}\left(\rho \overline{\mathrm{v}_{\mathrm{j}}}\right)\right]
\end{array}\right\} \mathrm{n}_{\mathrm{j}}=\mathrm{t}_{\mathrm{i}} \quad(\mathrm{i}, \mathrm{j}, \mathrm{k}=1,2,3) \text { in } \Gamma \mathrm{t}
\end{gathered}
$$

and the corresponding initial conditions:

$$
\begin{gathered}
\mathrm{v}_{\mathrm{i}}=\hat{\mathrm{v}}_{\mathrm{i} 0 \quad} \quad(\mathrm{i}, \mathrm{j}, \mathrm{k}=1,2,3) \text { in } \Omega \\
\mathrm{p}=\hat{\mathrm{p}}_{0} \quad \text { in } \Omega
\end{gathered}
$$

Where:

$$
\begin{aligned}
& \mathrm{L}_{\mathrm{ij}}=\overline{\overline{\mathrm{v}_{\mathrm{i}}}} \overline{\mathrm{v}_{\mathrm{j}}}-\overline{\mathrm{v}_{\mathrm{i}}} \overline{\mathrm{v}_{\mathrm{j}}}, \text { Leonard's terms } \\
& \mathrm{Cij}=\overline{\overline{\mathrm{v}_{\mathrm{i}}} \mathrm{v}_{\mathrm{j}}{ }^{\prime}}+\overline{\mathrm{v}_{\mathrm{i}}{ }^{\prime} \overline{\mathrm{v}_{\mathrm{j}}}}, \text { Crossed terms } \\
& \overline{\mathrm{v}_{\mathrm{i}}{ }^{\prime} \mathrm{v}_{\mathrm{j}}{ }^{\prime}}=\text { subgrid Reynolds stress components }
\end{aligned}
$$

The terms $\mathrm{L}_{\mathrm{ij}}$ and $\mathrm{C}_{\mathrm{ij}}$ can be neglected (Findikakis and Street, 1982). Previous studies (Petry and Awruch, 1997) confirm that the consideration of these terms had not significant influence and le to an increase of about $20 \%$ in the processing time.

Equations (2) and (3), neglecting the Leonard's and crossed terms, with the boundary and initial conditions given by Equations (4), (5), (6) and (7), are the governing equations of the turbulent, isothermal, quasi-incompressible flow of a Newtonian fluid. Adding the subgrid scale model equations, the system to be solved is obtained.

\section{Subgrid Scale Models}

The two-implemented models are based on the eddy viscosity concept. Using the hypothesis of Bousinesq, the subgrid Reynolds stresses are given by:

$$
\overline{-v_{i}{ }^{\prime} v_{j}{ }^{\prime}}=v_{t}\left(\frac{\partial \overline{v_{i}}}{\partial x_{j}}+\frac{\partial \overline{v_{j}}}{\partial x_{i}}\right)
$$

where $v_{\mathrm{t}}$ is the eddy viscosity.

This is the original Bousssinesq's equation. Usually, for incompressible flows, Equation (8) is modified (Hinze, 1975) by the introduction of a term with the subgrid kinetic energy to make the model compatible with the usual mass conservation equation for incompressible flows ( $\operatorname{div} \mathbf{v}=0$ ). However, in this work the continuity equation is the one modified for quasi-incompressible flows (Equation 2). Therefore, Equation (8) is used without any additional term.

\section{Smagorinsky's Model}

The model of Smagorinsky (1963) has been traditionally used to represent the effect of the subgrid scales in Large Eddy Simulation (Findikakis and Street, 1982; Lesieur et al, 1995). It is an eddy viscosity model in which the subgrid Reynolds stresses is given by Equation (8) and the eddy viscosity is defined as:

With the following boundary conditions:

$$
v_{\mathrm{t}}=\mathrm{Cs}^{2} \bar{\Delta}^{2}|\overline{\mathrm{S}}|
$$


where $\mathrm{C}_{\mathrm{S}}$ is the Smagorinsky's constant, with varying values from 0.1 to 0.22 , and the other terms are given by:

$$
\begin{gathered}
|\bar{S}|=\sqrt{2 \bar{S}_{i j} \bar{S}_{i j}} \\
\bar{S}_{i j}=\frac{1}{2}\left(\frac{\partial \bar{v}_{i}}{\partial x_{j}}+\frac{\partial \bar{v}_{j}}{\partial x_{i}}\right)
\end{gathered}
$$

The characteristic length $\bar{\Delta}$ is calculated as:

$$
\bar{\Delta}=\sqrt[3]{\prod_{\mathrm{i}=1}^{3} \Delta \mathrm{x}_{\mathrm{i}}}
$$

\section{Eddy Viscosity Dynamic Model}

The dynamic model was first proposed by Germano et al., 1991, and modified by Lilly, 1992. The subgrid Reynolds stresses are also obtained with Equation (8), but the eddy viscosity is defined as:

$$
v_{\mathrm{t}}=\mathrm{C}(\mathrm{x}, \mathrm{t}) \bar{\Delta}^{2}|\overline{\mathrm{S}}|
$$

The dynamic coefficient, $\mathrm{C}(\mathbf{x}, \mathrm{t})$, is calculated as a function of the local flow characteristics, using a double filtering process and it is based on information of the small scales solved by the mesh, defined as:

$$
C(x, t)=-\frac{1}{2} \frac{L_{i j} M_{i j}}{M_{i j} M_{i j}}
$$

where $\mathrm{L}_{\mathrm{ij}}$ e $\mathrm{M}_{\mathrm{ij}}$ are given by:

$$
\begin{gathered}
L_{i j}=\left\langle\bar{v}_{i} \bar{v}_{j}\right\rangle-\left\langle\bar{v}_{i}\right\rangle\left\langle\bar{v}_{j}\right\rangle \\
M_{i j}=\langle\bar{\Delta}\rangle^{2}|\langle\bar{S}\rangle|\left\langle\bar{S}_{i j}\right\rangle-\left\langle\bar{\Delta}^{2}\left|\bar{S}^{\prime}\right| \bar{S}_{i j}\right\rangle
\end{gathered}
$$

with

$$
\begin{gathered}
\left\langle\bar{S}_{\mathrm{ij}}\right\rangle=\frac{1}{2}\left(\frac{\partial\left\langle\overline{\mathrm{v}}_{\mathrm{i}}\right\rangle}{\partial \mathrm{x}_{\mathrm{j}}}+\frac{\partial\left\langle\overline{\mathrm{v}}_{\mathrm{j}}\right\rangle}{\partial \mathrm{x}_{\mathrm{i}}}\right) \\
|\langle\overline{\mathrm{S}}\rangle|=\sqrt{\left.2\left\langle\overline{\mathrm{S}}_{\mathrm{ij}}\right\rangle \overline{\mathrm{S}}_{\mathrm{ij}}\right\rangle}
\end{gathered}
$$

$\langle\bar{\Delta}\rangle$ is the characteristic length of the second filter, larger than the characteristic length of the first filter $-\langle\bar{\Delta}\rangle>\bar{\Delta}$.

In the above equations, the bar indicates the first filtering process (filter at mesh level) and the symbol \langle\rangle indicates the second filtering process (test filter).

For the solution of the system of equations, the Finite Element Method is employed. To get the system of algebraic equations, time derivatives are expanded in Taylor series, including the second order terms. For the space discretization the classic Galerkin method is applied (Reddy and Gartling, 1994). To save processing time, analytical expressions for the hexaedrical isoparametric element matrices are used (Burbridge and Awruch, 2000). This scheme is known as Taylor-Galerkin, (Donea, 1984), and was used by Azevedo, 1999, for the simulation of three-dimensional laminar flows with fluid-structure interaction. The scheme is explicit and conditionally stable and the integration time step has the following restriction:

$$
\Delta \mathrm{t} \leq \frac{\Delta \mathrm{x}_{\mathrm{i}}(\min )}{\mathrm{C}+\mathrm{V}}
$$

where $\Delta \mathrm{x}_{\mathrm{i}}(\mathrm{min})$ is the minimum dimension of the mesh elements, $\mathrm{C}$ is the speed of sound and $\mathrm{V}$ is the reference velocity.

\section{The Second Filter: Proposed Methodology}

Equation (14) defines the dynamic coefficient, $\mathrm{C}(\mathbf{x}, \mathrm{t})$. This coefficient depends on the use of two filters of different characteristic lengths. The first filter, at the mesh level, has characteristic length related to the element dimension. For the second filtering process (test filter) the characteristic length must be greater than the length of the first filter.

For the second filtering process many proposals have been presented. Oshima, et al., 1996, formulate the second filtering operation in a Finite Element code using expansions in Taylor series. Padilla and Silveira Neto, 2000, present and compare different methodologies in the context of Finite Volumes.

The new methodology used in this work was presented in Petry, 2002. The Second Filter by Independent Finite Elements uses techniques common to finite elements, such as: the definition of elements by conectivities; the use of two systems of coordinates (global $\left(\mathrm{x}_{1}, \mathrm{x}_{2}, \mathrm{x}_{3}\right)$ and natural $(\xi, \eta, \zeta)$ ); the transformations of coordinates and elements interpolation functions. The scheme consists on generating one super-element around each node of the mesh, then, with the usual finite element shape functions, a linear interpolation of the variables at the super-element nodes is performed to get filtered values in the corresponding internal node. This procedure is organized in three stages.

The first stage is included in the pre-processing phase (mesh generation), and consists of generating a list of conectivities of the independent super-elements for each node. In this scheme the test filter dimension is not prescribed, but it is possible to include some restriction with respect to such dimension. In Figure 1 an example of an independent super-element, with its corresponding internal node, is presented.

The second stage, also included in the pre-processing phase to reduce additional computational cost, consists of the evaluation of the natural coordinates $\left(\xi_{I}, \eta_{I}, \zeta_{I}\right)$ of node $I$ in the interior of its independent super-element. Global coordinates of a point I inside of an element can be calculated by the following transformation of coordinates:

$$
\left(x_{i}\right)_{I}=\sum_{\alpha=1}^{8} \Psi_{\alpha}\left(\xi_{I}, \eta_{I}, \zeta\right) x_{i \alpha}
$$

This is the usual transformation used in finite elements, where $\left(\mathrm{x}_{\mathrm{i}}\right)_{\mathrm{I}}$ is the coordinate of any point I inside an independent superelement; $\mathrm{X}_{\mathrm{i} \alpha}$ is the coordinate of node $\alpha,(\alpha=1,2,3,4,5,6,7,8)$, (i=1, 2, 3) and $\psi_{\alpha}\left(\xi_{\mathrm{I}}, \eta_{\mathrm{I}}, \zeta_{\mathrm{I}}\right)$ is the interpolation function of node $\alpha$, evaluated at point I, with natural coordinates $\left(\xi_{I}, \eta_{I}, \zeta_{I}\right)$. 


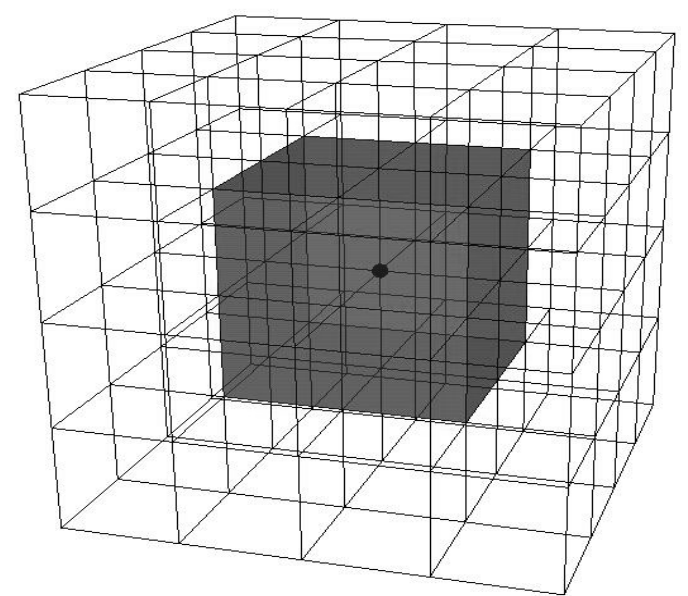

Figure 1. Independent Super-Element created around a node, to be used in the second filtering process.

The interpolation functions for an isoparametric eight node hexahedrical element are given by:

$$
\left\{\Psi^{e}\right\}=\frac{1}{8}\left\{\begin{array}{l}
(1-\xi)(1-\eta)(1-\zeta) \\
(1+\xi)(1-\eta)(1-\zeta) \\
(1+\xi)(1+\eta)(1-\zeta) \\
(1-\xi)(1+\eta)(1-\zeta) \\
(1-\xi)(1-\eta)(1+\zeta) \\
(1+\xi)(1-\eta)(1+\zeta) \\
(1+\xi)(1+\eta)(1+\zeta) \\
(1-\xi)(1+\eta)(1+\zeta)
\end{array}\right\}
$$

It is necessary to solve the inverse problem represented by Equation (18), to obtain the natural coordinates of a point inside the element $\left(\xi_{\mathrm{I}}, \eta_{\mathrm{I}}, \zeta_{\mathrm{I}}\right)$, from its global coordinates, $\left(\mathrm{x}_{1}, \mathrm{x}_{2}, \mathrm{x}_{3}\right)_{\mathrm{I}}$, and the global coordinates of the eight nodes of the super-element, $\left(\mathrm{x}_{1}, \mathrm{x}_{2}, \mathrm{x}_{3}\right)_{\alpha}$. In the analysis of this problem, a non-linear system with three equations is derived and an algorithm using an iterative process of solution was implemented to solve the system. Using the Newton iterative method, the following system is obtained:

$$
\mathbf{U}^{\mathbf{n}+\mathbf{1}}=\mathbf{U}^{\mathbf{n}}-\mathbf{J}^{-1}\left(\mathbf{U}^{\mathrm{n}}\right) \mathbf{R}\left(\mathbf{U}^{\mathbf{n}}\right)
$$

where

$$
\mathbf{R}(\mathbf{U})=\sum_{\alpha=1}^{8} \Psi_{\alpha}\left(\xi_{\mathrm{I}}, \eta_{\mathrm{I}}, \zeta_{\mathrm{I}}\right) \mathrm{x}_{\mathrm{i} \alpha}-\left(\mathrm{x}_{\mathrm{i}}\right)_{\mathrm{I}}
$$

and the jacobian matrix, $\mathbf{J}\left(\mathbf{U}^{\mathrm{n}}\right)$, is given by

$$
J=\left[\begin{array}{lll}
\frac{\partial R_{1}}{\partial \xi} & \frac{\partial R_{1}}{\partial \eta} & \frac{\partial R_{1}}{\partial \zeta} \\
\frac{\partial R_{2}}{\partial \xi} & \frac{\partial R_{2}}{\partial \eta} & \frac{\partial R_{2}}{\partial \zeta} \\
\frac{\partial R_{3}}{\partial \xi} & \frac{\partial R_{3}}{\partial \eta} & \frac{\partial R_{3}}{\partial \zeta}
\end{array}\right]
$$

The solution vector $\mathbf{U}$, contains the natural coordinates of the point I , $\left(\xi_{\mathrm{I}}, \eta_{\mathrm{I}}, \zeta_{\mathrm{I}}\right)$, at the interior of the super element.
Only the last stage is computed in the main code. It consists of the evaluation of the second filtered values at each node, by a finite element interpolation function inside the independent superelements, calculated by the following expressions:

$$
\begin{gathered}
\left\langle\overline{\mathrm{v}}_{\mathrm{i}}\right\rangle_{\mathrm{I}}=\sum_{\alpha=1}^{8} \psi_{\alpha}\left(\xi_{\mathrm{I}}, \eta_{\mathrm{I}}, \zeta_{\mathrm{I}}\right) \overline{\mathrm{v}}_{\mathrm{i} \alpha} \\
\left\langle\overline{\mathrm{v}}_{\mathrm{i}} \overline{\mathrm{v}}_{\mathrm{j}}\right\rangle_{\mathrm{I}}=\sum_{\alpha=1}^{8} \psi_{\alpha}\left(\xi_{\mathrm{I}}, \eta_{\mathrm{I}}, \zeta_{\mathrm{I}}\right)\left(\overline{\mathrm{v}}_{\mathrm{i}} \overline{\mathrm{v}}_{\mathrm{j}}\right)_{\alpha} \\
\left\langle\bar{\Delta}^{2}|\overline{\mathrm{S}}| \overline{\mathrm{S}}_{\mathrm{ij}}\right\rangle_{\mathrm{I}}=\sum_{\alpha=1}^{8} \psi_{\alpha}\left(\xi_{\mathrm{I}}, \eta_{\mathrm{I}}, \zeta_{\mathrm{I}}\right)\left(\bar{\Delta}^{2}|\overline{\mathrm{S}}| \overline{\mathrm{S}}_{\mathrm{ij}}\right)_{\alpha}
\end{gathered}
$$

The characteristic dimension of the test filter is calculated in the same way as for the first filter, given by Equation (12), but considering the dimensions of the independent super-elements.

As the eddy viscosity, given by Equation (13), is evaluated at the element level and the values of the dynamic coefficient, given by Equation (14), are calculated for each node of the mesh, the coefficient used for each element is the average of the nodal values of $\mathrm{C}(\mathbf{x}, \mathrm{t})$. This procedure is in accordance with other authors (Oshima et al, 1996; Zang et al, 1993, Breuer and Rodi, 1994), that uses averages of $\mathrm{C}(\mathbf{x}, \mathrm{t})$ to prevent abrupt variations in space and time, wich are sources of instabilities in the numerical solution. Another technique, cited by Lilly, 1992, consists of proceeding averages of the terms $\mathrm{M}_{\mathrm{ij}}$ and $\mathrm{L}_{\mathrm{ij}}$ (Equations 15) before the calculation of $\mathrm{C}(\mathrm{x}, \mathrm{t})$, stabilizing the problem and preventing zeros in the denominator.

In this work, a limit for negative values of the eddy viscosity was adopted, which is expressed in Equation (26). Zang et al, 1993, used the same limit.

$$
v+v_{t} \geq 0
$$

Another procedure adopted here is that if $\mathrm{M}_{\mathrm{ij}}$ is equal to zero, it is assumed that $\mathrm{C}(\mathbf{x}, \mathrm{t})=0$, in the corresponding node.

The dynamic model increases the total processing time between 9 and $18 \%$, in comparison to the classical Smagorinsky's model, for the problems analyzed in this work.

\section{Numerical Examples}

\section{Backward-Facing Step}

Flow simulations of a two-dimensional backward-facing step with low Reynolds numbers, were initially performed in order to validate the code. The results were obtained using the Smagorinsky's model and the dynamic model, and compared with experimental data (Armaly et al., 1983) and other numerical simulations (Silveira Neto et al., 1993; Kaiktsis et al, 1991).

The problem domain is presented in Figure 2. For the twodimensional case, there is only one element in the $\mathrm{z}$ direction, and the components of the velocity in this direction are equal to zero $\left(v_{3}=0\right)$ over the whole flow field. The dimensions are similar to the experimental work presented by Armaly et al, 1983.

For problems characterized by Reynolds number less than or equal to 1000 , simulations are long enough to reach the stationary time average flow.

As inflow boundary conditions, a completely developed parabolic velocity profile was used $\left(\mathrm{v}_{1}=\mathrm{v}(\mathrm{y}), \mathrm{v}_{2}=0\right)$ at the entrance. The non-slip condition $\left(\mathrm{v}_{1}=\mathrm{v}_{2}=\mathrm{v}_{3}=0\right)$ was prescribed at the uppe and lower walls. At the outflow, natural boundary conditions were 
employed $\left(\mathrm{t}_{1}=\mathrm{t}_{2}=\mathrm{t}_{3}=0\right)$ (Equation 5). Homogeneous initial conditions $\left(\mathrm{v}_{1}=\mathrm{v}_{2}=\mathrm{v}_{3}=\mathrm{p}=0\right)$ were used, in the first simulation, for $\mathrm{Re}=100$. In the other simulations the last fields of pressures and velocities calculated for previous Reynolds number were used.

The Reynolds number is defined in the same way that in the experiments of Armaly et al., 1983, with $\mathrm{h}=1.0 \mathrm{~m}$.

$$
\operatorname{Re}=\frac{\rho\left(2 \mathrm{~V}_{\max } / 3\right) 2 \mathrm{~h}}{\mu}
$$

The maximum velocity value at the entrance, $V_{\max }$ is of $75 \mathrm{~m} / \mathrm{s}$. The dimensionless reattachment length is defined as $\mathrm{X}_{\mathrm{r}} / \mathrm{H}$, where $\mathrm{H}$ is height of the step $(0.94 \mathrm{~m})$.

To obtain the reattachment length it was investigated the first layer of nodes above the lower wall behind the step. The reattachment point is defined as the first node of the mesh, after the separation region (Figure 2), where the component $\mathrm{v}_{1}$ of the average velocity field assumes a positive value. The results for the relation $\mathrm{X}_{\mathrm{r}} / \mathrm{H} \times \mathrm{Re}$ that was obtained in the present work, together with the values presented in Armaly, et al., 1983, are shown in Table 1.

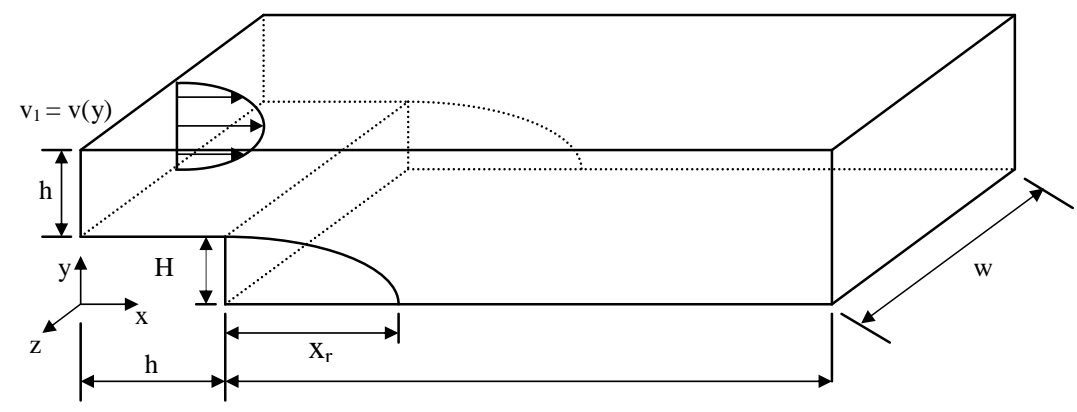

Figure 2. Domain and characteristics dimensions of the backward-facing step.

The results for $\mathrm{Re}=100$ and $\mathrm{Re}=400$ are close to those obtained experimentaly, for both models. For laminar flows no important differences between results were expected. The dynamic model for $\mathrm{Re}=100$ presented $95 \%$ of the nodes with $\mathrm{C}(\mathbf{x}, \mathrm{t})$ equal to zero, the flow with $\mathrm{Re}=400$ presented $94 \%$ of the nodes with $\mathrm{C}(\mathbf{x}, \mathrm{t})$ equal to zero and there were no elements with negative eddy viscosity. For the flow with $\mathrm{Re}=1000,60 \%$ of the nodes presented dynamic coefficient equal to zero, while $0.09 \%$ of the elements had negative eddy viscosity, where Equation (26) was applied.

Table 1. Reattachment length , $\mathrm{Xr} / \mathrm{H}$, as a function of the Reynolds number: present work $x$ experimental values (Armaly et al, 1995).

\begin{tabular}{|c|c|c|c|}
\hline \multicolumn{4}{|c|}{ Reattachment Length $-\mathbf{X}_{\mathbf{r}} / \mathbf{H}$} \\
\hline Re & $\begin{array}{c}\text { Smagorinsky's } \\
\text { Model }\end{array}$ & $\begin{array}{c}\text { Dynamic } \\
\text { Model }\end{array}$ & Armaly et al. 1983 \\
\hline 100 & 2.74 & 2.89 & 3.0 \\
\hline 400 & 7.60 & 7.90 & 8.0 \\
\hline 1000 & 11.09 & 11.25 & 16.0 \\
\hline
\end{tabular}

For $\mathrm{Re}=1000$, the numerical value is far from the experimental results. This was an expected error, because in two-dimensional simulations of laminar flows, with Reynolds numbers larger than 500 , the reattachment length is underestimated. The error is due to three-dimensional effects, neglected in two-dimensional simulations (Silveira Neto et al, 1993, and Kaiktsis et al, 1991) and verified against the numerical experiments of Williams and Baker, 1997.

To simulate a turbulent flow, $\mathrm{Re}=10,000$ was used. At the time instant of $0.4 \mathrm{~s}$, using the dynamic model, the dimensionless reattachment length was of 7.3, whereas experimental values vary between 6 and 8 (Kim et al, 1980). The number of elements where negative eddy viscosity values were found was less than $6 \%$.

Finally, three-dimensional flows over a backward facing step, without sidewalls, for Reynolds number 100 and 1,000, were simulated using the dynamic model. The problem domain is presented in Figure 2, where $w=2 \mathrm{~m}$. The adopted mesh is similar to that employed for the previous two-dimensional simulations, but with eight elements in the $\mathrm{Z}$ direction. Boundary conditions are similar to those used in the two-dimensional case. In the first simulation $(\mathrm{Re}=100)$ homogeneous initial conditions were used and in the following simulation $(\mathrm{Re}=1,000)$ the last fields of pressures and velocities calculated for the Reynolds number 100 are used as initial conditions.

As for the two-dimensional example with $\mathrm{Re}=100$, the obtained reattachment length was equal to 2.88 . For the flow with $\mathrm{Re}=1,000$, instantaneous results can be observed in Figures 3 and 4. In the simulations of the backward facing step, spurious oscillations of pressure were observed and were controlled by reducing the time step, conducting to excessive processing time, than the statistical stationary state of this case was not performed.

Despite the low resolution in the $\mathrm{Z}$ direction, where only eight elements were taken, the results are qualitatively coherent with the results found in other references (Silveira Neto et al., 1993; Lesieur, 1999 and Williams and Baker, 1997). Figures 3 and 4 show that the flow is developed as expected.

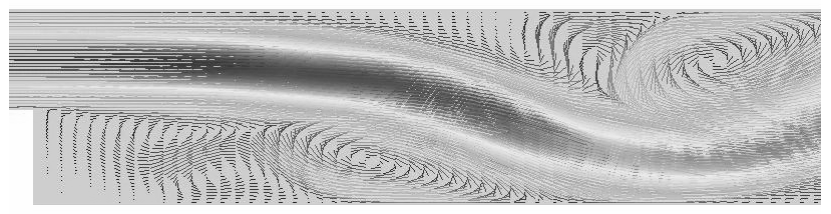

Figure 3. Detail of the velocity vectors field, in the mainstream direction at $z=1.0 \mathrm{~m}$, for the three-dimensional case with the dynamic model and $\mathrm{Re}=1,000$.

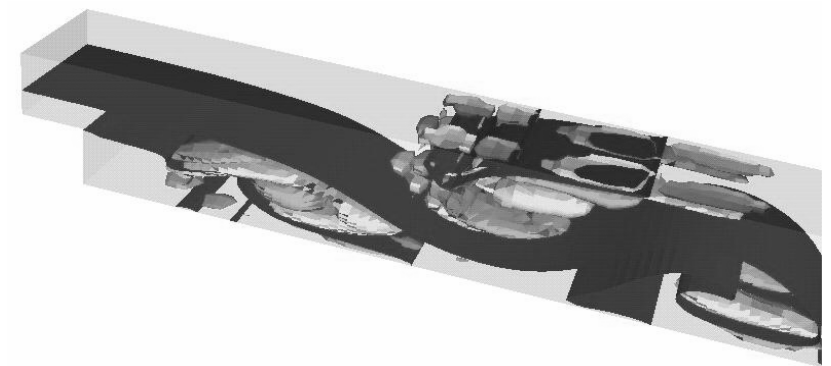

Figura 4. Vorticity field (w), for $R e=1,000$, using the dynamic model: $w_{3}=5.7 \times 10^{1},-w_{1}=4 \times 10^{-4} e-w_{1}=-4 \times 10^{-4}$. 


\section{The Driven Cavity Flow}

This section presents results of three-dimensional driven cavity flow simulations for Reynolds numbers of 3,200 and 10,000. This is an interesting flow, with a complex behavior in a simple geometry (Figure 5), and was investigated numerically (Denaro, 1996; Kim and Menon, 1999; Zang et al., 1993) and experimentally (Prasad and Koseff, 1989) by many researchers. The experimental results of Prasad and Koseff, 1989, were used to compare with the present numerical study. The obtained results are close to the experimental results and have comparable quality to the numerical analysis developed by of Zang, Street and Koseff, 1993, and Kim and Menon, 1999.

The aspect ratio of the cavity flow characterized by $\mathrm{Re}=3,200$ is $1(1: 1: 1)$ while the cavity flow characterized by $\operatorname{Re}=10,000$ has aspect ratio of 0.5 (1:1:0.5). The Reynolds number is calculated based on the velocity of the upper wall $\mathrm{U}_{\mathrm{B}}$ (Figure 5), which moves and induces the flow. All others walls are fixed, with non-slip boundary condition.

Numerical simulations for the complete and the half domain considering symmetry were developed for $R e=3,200$, with Smagorinsky's model (Petry, 2002). Results obtained for average velocity profiles, as well as the statistics of turbulence, were very similar for both cases, so, to reduce processing time, it is considered that the flow has a symmetry plane in $\mathrm{H} / 2$ (Figure 5) for simulations presented here.

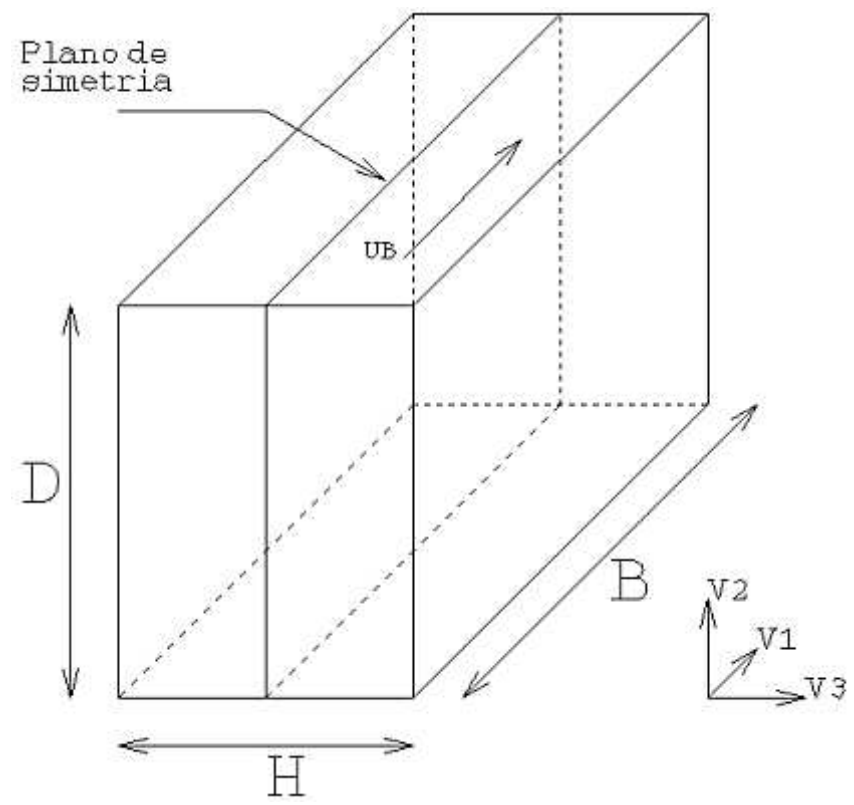

Figure 5. Geometry of the driven cavity flow, similar of that used by Prasad and Koseff, 1989.

\section{Three-Dimensional Cavity Flow: $\mathbf{R e}=\mathbf{3 2 0 0}$}

This section presents results for three-dimensional cavity flow characterized by $\mathrm{Re}=3200$ and an aspect ratio equal to 1 $(B=1: D=1: H=1)$, assuming a symmetry plane (Figure 5). The velocity $U_{B}$ is equal to $100 \mathrm{~m} / \mathrm{s}$, the sound speed, $C$, is equal to $340 \mathrm{~m} / \mathrm{s}$ and the time step is $\Delta \mathrm{t}=1.5 \times 10^{-5} \mathrm{~s}$. A three-dimensional view of the mesh is presented in Figure 6. The mesh has $32 \times 32$ elements in $\mathrm{x}$ and a $\mathrm{y}$ direction, refined near the walls, and has 16 elements in the $\mathrm{z}$ direction. The dimension of the smallest element edge is of the order of $1 \times 10^{-2}$.

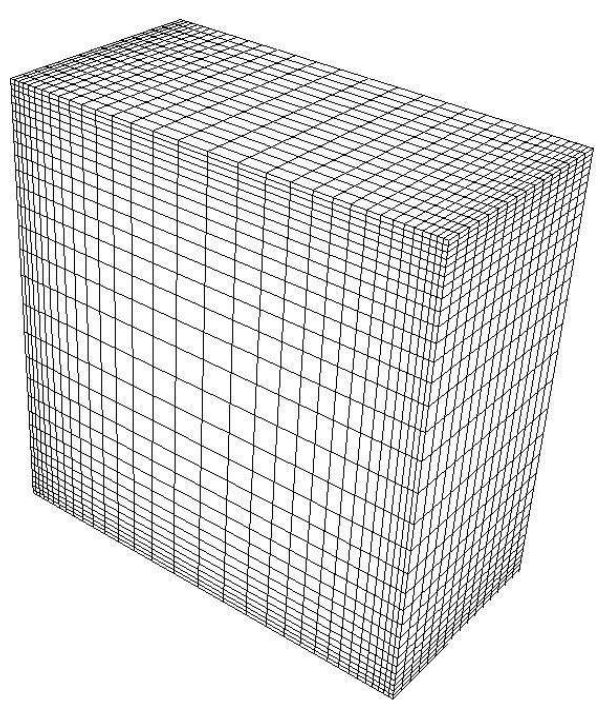

Figure 6. Finite element mesh for the 3D cavity flow, $R e=3200$.

Data for the statistical analysis of turbulence were obtained storing all variables along the centerlines at horizontal and vertical directions at the symmetry plane, for each time interval. The flow was analyzed until $0.795 \mathrm{~s}$, but for the statistical analysis it was considered the final $0.24 \mathrm{~s}$ period. The experimental values presented were taken from the figures published by Prasad and Koseff, 1989.

The dimensionless mean velocities presented in Figures 7 and 11 are defined as:

$$
\begin{aligned}
& \mathrm{V} 1=\frac{\overline{\mathrm{v}_{1}}}{\frac{\mathrm{U}_{\mathrm{B}}}{\overline{\mathrm{v}_{2}}}} \\
& \mathrm{~V} 2=\frac{\mathrm{U}_{\mathrm{B}}}{\mathrm{U}_{\mathrm{B}}}
\end{aligned}
$$

where the double bar indicates time average. Dimensionless forms of the root mean square, V1rms and V2rms and for Reynolds stresses UV1 and UV2 are defined in the same way as published by other authors (Zang et al., 1993 and Prasad and Koseff, 1989):

$$
\begin{gathered}
\text { V1rms }=10 \sqrt{\overline{\frac{\mathrm{v}_{1}^{\prime \prime 2}}{\mathrm{U}_{\mathrm{B}}^{2}}}} \\
\mathrm{~V} 2 \mathrm{rms}=10 \sqrt{\frac{\overline{\frac{\mathrm{v}_{2}^{\prime \prime 2}}{\mathrm{U}_{\mathrm{B}}^{2}}}}{\bar{U}}} \\
\mathrm{UV1}=\mathrm{UV} 2=500 \frac{\mathrm{v}_{1}^{\prime \prime} \mathrm{v}_{2}^{\prime \prime}}{\mathrm{U}_{\mathrm{B}}^{2}}
\end{gathered}
$$

where:

$$
\mathrm{v}_{1}^{\prime \prime}=\mathrm{v}_{1}-\overline{\overline{\mathrm{v}_{1}}} \quad \mathrm{v}_{2}{ }^{\prime \prime}=\mathrm{v}_{2}-\overline{\overline{\mathrm{v}_{2}}}
$$

Three simulations were developed for this Reynolds number the first one using a no model code - Laminar - and the others using the the two subgrid models codes - Smagorinsky and Dynamic. 
Results for the mean velocity components are coincident for the three simulations developed for this Reynolds number and have good agreement with the experimental data, as it is possible to observe in Figure 7. Figures 7, 8 and 9 show results obtained in the present work as compared with the published experimental results of Prasad and Koseff, 1989.

The distributions of the correlations of the velocity fluctuations, presented in Figures 8 and 9, are very close to results of Prasad and Koseff, 1989. The peak of the Reynolds stresses (UV1) near the bottom wall is more accurately captured by the dynamic model as can be verified in Figure 9. The obtained profiles clearly contain three-dimensional effects, in view of differences observed in 2D and 3D cavity flows, as presented by Prasad and Koseff, 1989.

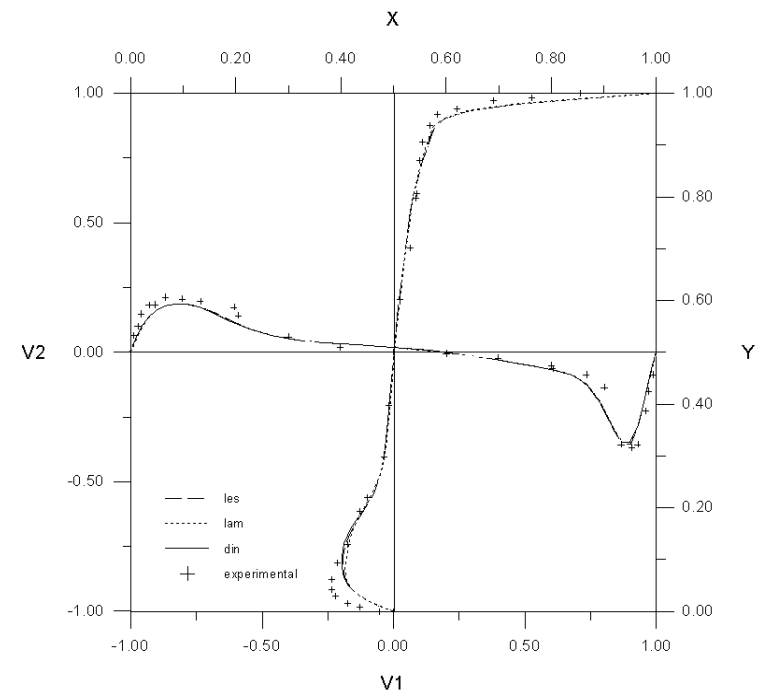

Figure 7. Dimensionless mean velocity components (Eq 28) profiles at center lines at the symmetry plane, $R e=3200:+-$ experimental results [Prasad e Koseff, 1989]; - - - Smagorinsky; . . . .Laminar and Dynamic models.

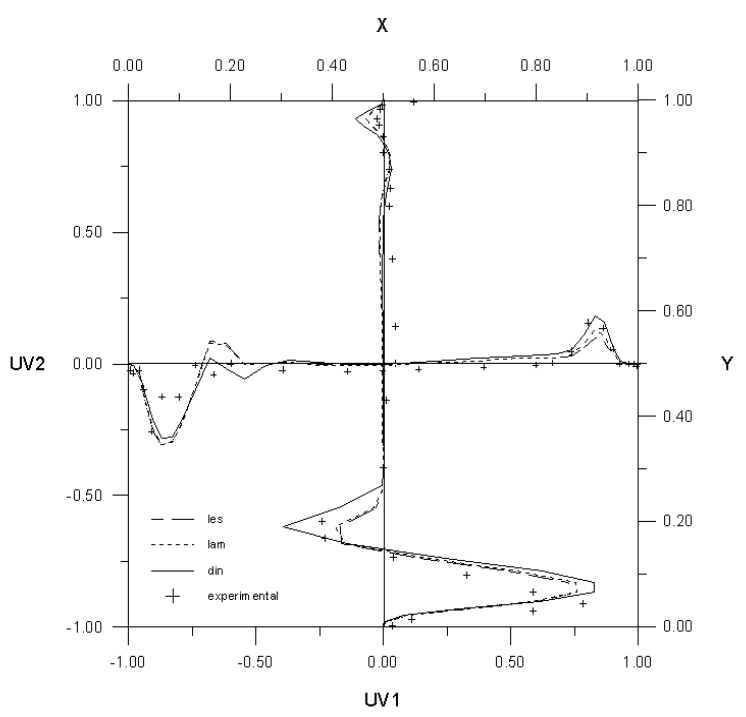

Figure 9. Dimensionless Reynolds Stresses (Eq 30) profiles at center lines at the symmetry plane, $R e=3200:+-$ experimental results [Prasad $e$ Koseff, 1989];--- Smagorinsky; . . . . . Laminar and

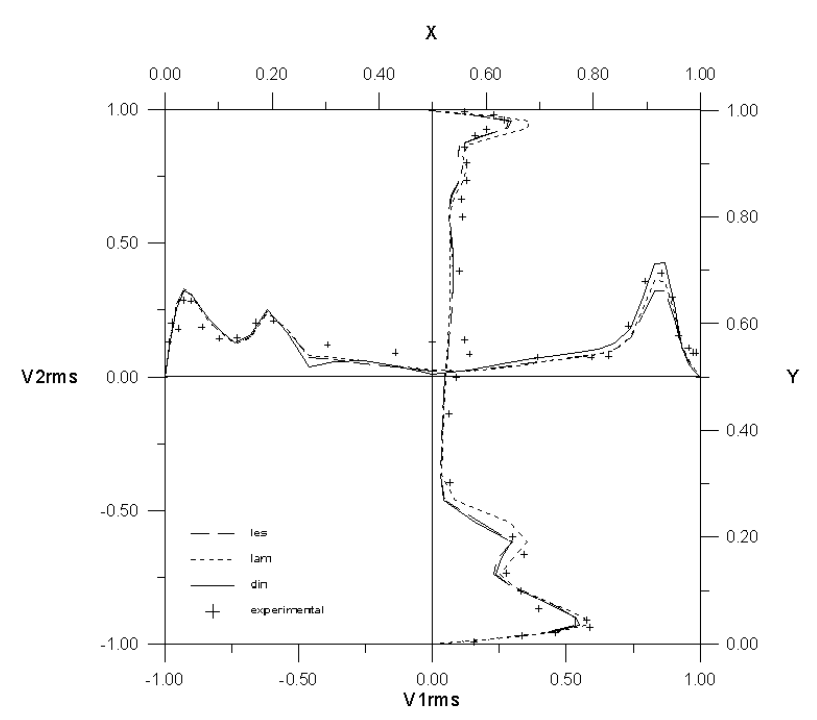

Figure 8. Dimensionless velocity root mean square components (Eq. 29) profiles at center lines at the symmetry plane, $R e=3200$ : + - experimental results [Prasad e Koseff, 1989]; - - - Smagorinsky; . . . . Laminar and Dynamic models.

\section{Three-Dimensional Cavity Flow: $\mathrm{Re}=\mathbf{1 0 , 0 0 0}$}

Results for a three-dimensional cavity flow characterized by $\mathrm{Re}=10,000$ and an aspect ratio equal to $0.5(\mathrm{~B}=1: \mathrm{D}=1: \mathrm{H}=0.5)$, assuming a symmetry plane (Figure 5 ), are presented in this section. The velocity $U_{B}$ is equal to $100 \mathrm{~m} / \mathrm{s}$, the sound speed, $C$, is equal to $340 \mathrm{~m} / \mathrm{s}$ and time is $\Delta \mathrm{t}=0.75 \times 10^{-5 \mathrm{~s}}$. The three-dimensional image of the mesh is presented in Figure 10. The mesh is uniform in the $\mathrm{z}$ direction and it is refined near walls in the $\mathrm{x}$ and $\mathrm{y}$ directions. The dimension of the $\mathrm{x}$ and $\mathrm{y}$ edge of the smallest element is of the order of $5 \times 10^{-3} \mathrm{~s}$.

Two simulations were developed for this Reynolds number, using the two subgrid models codes - Smagorinsky and Dynamic. No simulation was developed with the Laminar (no model) code, due to the high Reynolds value.

Data for the statistical analysis of turbulence were obtained storing the values of all variables corresponding to the centerlines in the horizontal and vertical directions at the symmetry plane, for each time interval. The flow was analysed until $1.095 \mathrm{~s}$ but for the statistical analysis it was considered the final 0.6265 s period. Figure 11 present dimensionless average velocity components (V1 and V2) as defined in Equation (28) at the centerlines of the symmetry plane. Figures 12 and 13 present the dimensionless mean square root values (V1rms, V2rms), defined in Equations (29), and the dimensionless Reynolds Stresses (UV1 and UV2), defined in the Equation (30).

The results of the present work are compared with experimental values published by Prasad and Koseff, 1989. The mean velocity results for this Reynolds number are very close to experimental results, as shown in Figure 11, for both models. In the correlations of turbulence there are differences, favorable to the dynamic model, as for example the inferior peaks, near the bottom wall of Reynolds stresses, UV1, (Figure13) and mean root square, V1rms (Figure 12). 


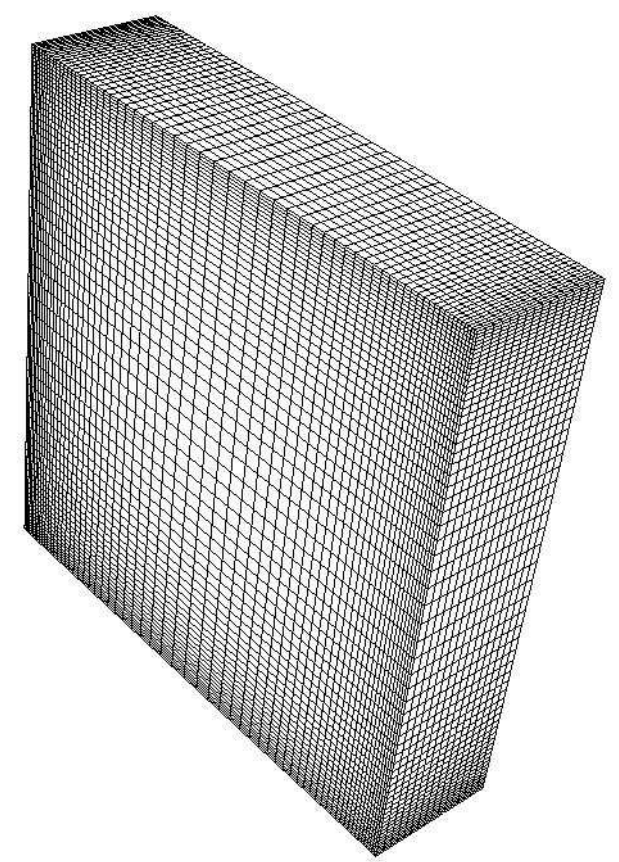

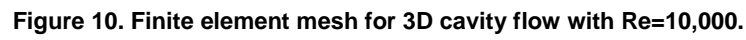

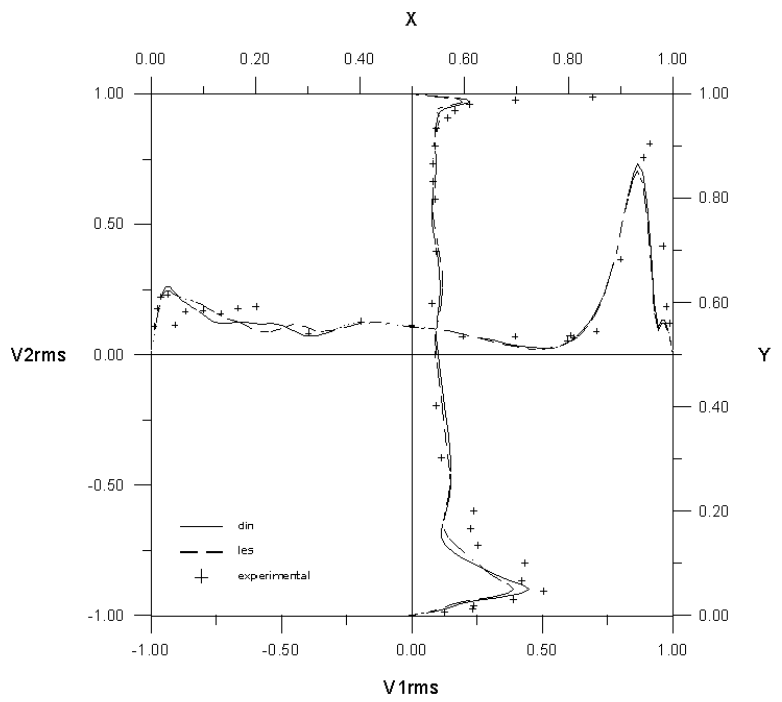

Figure 12 - Dimensionless velocity root mean square (Eq. 29) of components profiles at center lines at the symmetry plane, $R e=10,000:+-$ experimental results [Prasad e Koseff, 1989]; - - - Smagorinsky; and Dynamic models.

Other experiments are necessary to investigate the differences between the models. The numerical results are comparable with other numerical simulations published by Zang, et al, 1993, and confirms the good quality of present results.

Figure 11. Dimensionless mean velocity components (Eq. 28) profiles at center lines at the symmetry plane, $R e=10,000:+-$ experimental results [Prasad e Koseff, 1989]; - - - Smagorinsky; and __ Dynamic models.

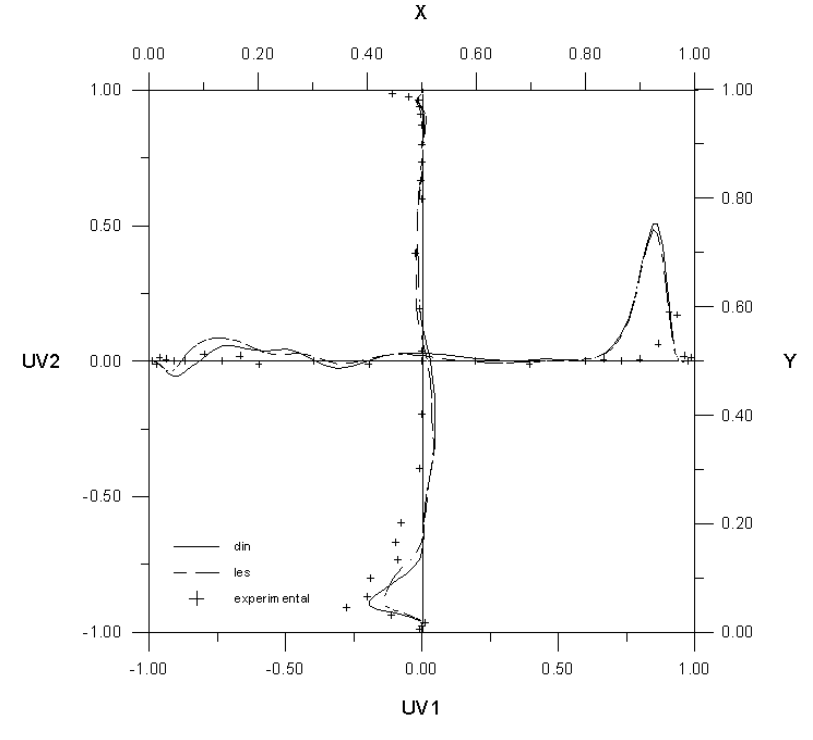

Figure 13. Dimensionless Reynolds Stresses (Eq. 30) profiles at center lines at the symmetry plane, $R e=10,000:+-$ experimental results [Prasad $e$ Koseff, 1989]; - . - Smagorinsky and Dynamic models.

\section{Conclusions}

The three dimensional classical problems of backward facing step and driven cavity flows were simulated with a Finite Element Large Eddy Simulation methodology presented in this work. Two subgrid scale models were implemented, the Smagorinsky's and the eddy viscosity dynamic model. The results of both models are coherent with experimental and numerical data from other authors. In the simulations of the backward facing step, spurious oscillations of pressure were observed and were controlled by reducing the time step, this procedure results in additional processing time that is critical in Large Eddy Simulations. For the cavity flow problem, statistical analysis of turbulence were included and the obtained 
values were close to other published results, not requiring any reduction in the time step for simulation.

A new scheme for the second filtering operation in the dynamic model was presented and applied. The scheme consists of defining super-elements around each node of the original mesh and applying the finite element interpolation functions to obtain the filtered quantities for the nodes. Most of this filtering operation is developed in the pre-processing, resulting in a dynamic model with total additional cost in processing time between 9 and $18 \%$, when compared to the implemented Smagorinsky's model. This additional time is in the same order of the best results reported by other authors.

Analysis of the problems presented here demonstrated the capability of this methodology to simulate complex turbulent flows, without restrictions about memory allocation. The long time of processing, resulting mostly from the time interval restriction of this explicit scheme, indicates the relevance to improve the code before continuing the simulation of other problems in view of extended analysis of different models behavior. Researches on these aspects are in progress.

\section{Acknowledgements}

The authors thank the National Supercomputer Center on the Federal University of Rio Grande do Sul.

\section{References}

Armaly, B.F., Durst, F., Pereira, J.C.F., Schönung, B., 1983 "Experimental and Theoretical Investigation of Backward-Facing Step Flow", Journal of Fluid Mechanics, Vol.127, pp. 473-496

Awruch, A.M., Petry, A.P., 1997. "On the Analysis of Viscous Incompressible Flows Using Finite Elements with the Primary Variables" (in Portuguese), Research Report - RP 122/97, . Programa de Pós-Graduação em Engenharia Civil, Universidade Federal do Rio Grande do Sul, Porto Alegre, RS, Brazil, 73 pages.

Azevedo, R. L. 1999. "Analysis of Fluid-Structure Interaction Problems Using the Finite Element Methot and Monolithic Coupling"(in Portuguese). DSc Thesis . Programa de Pós-Graduacão em Engenharia Civil, Universidade Federal do Rio Grande do Sul, Porto Alegre, RS Brazil

Breuer,M., Rodi,W., 1994. "Large Eddy Simulation of Turbulent Flow through a Straight Square Duct and $180^{\circ}$ Bend", Direct and Large Eddy Simulation I, pp. 273-285, P.R.Voke et al (eds.), Kluwer Academic Publishers, Netherlands.

Burbridge, H.P., Awruch, A.M., 2000. An efficient one-step TaylorGalerkin Scheme to Analyze 3D High Compressible Flows Using the Finite Element Method, Anais ENCIT 2000, Porto Alegre, RS,Brasil

Denaro, F. M., 1996. "Towards a new Model-Free Simulation of HighReynolds-Flows: Local Average Direct Numerical Simulation”. Int. J. Num. Meth. Fluids., Vol.23, pp. 123-142.

Donea, J, 1984. "A Taylor-Galerkin Method for Convective Transport Problems", International Journal for Numerical Methods in Engineering, Vol.20, pp. 101-119

Ferziger, J.H.,1993. "Simulation of complex turbulent flow: recent advances and prospects in wind engineering", Journall of Wind Engineering and Industrial Aerodynamics, Vol.46 \& 47, pp. 195-212, Elsiever.

Findikakis, A.N.; Street, R.L., 1982. "Mathematical Description of Turbulent Flows", Journal of Hydraulics Division, ASCE, Vol.108, No.HY8, paper 17265 , pp. 887-903.

Franca L.P.; Frey, S.1992. Stabilized Finite Element Method: II. The incompressible Navier-Stokes equations. Comp. Meth. Appl. Mech. Eng. Vol.99, pp. 209-233.
Germano, M. Piomelli, U., Moin, P. CabotW.H., 1991. “A dynamic subgrid-scale eddy viscosity model”, Phys.Fluids A3 (7), pp. 1760-1765. Hinze, J.O., 1975. Turbulence, McGraw-Hill, New York, 2nd ed.

Hughes, T. J. R., 1987. The Finite Element Method, Prentice-Hall, New Jersey.

Kaiktsis, L., Karniadakis, G., Orzag, S.A., 1991. "Onset of threedimensionality, equilibria, and early transition in flow over a BackwardFacing Step", Journal of Fluid Mechanics, Vol..231, pp. 501-528.

Kawahara, M., Hirano, H.,1983. "A Finite Element Method for High Reynolds Number Viscous Fluid Flow Using Two Step Explicit Scheme". International Journal for Numerical Methods in Fluids, Vol.3, pp. 137-163.

Kim, J., Kline, S.J., Jhonston, J.P., 1980. Investigation of a reattaching turbulent shear Layer flow over a backward-facing step. ASME J.Fluids Engng., Vol.102, pp. 302-308.

Kim, W., Menon, S., 1999. "An Unsteady Incompressible Navier-Stokes Solver for Large Eddy Simulation of Turbulent Flows". Int. J. Numer. Meth. Fluids, Vol.31, pp. 983-1017.

Lesieur, M. Comte, P., Métais, O., 1995. "Numerical simulations of coherent vortices in turbulence", ASME - Appl Mech Rev, Vol. 48, No.4.

Lilly, D.K., 1992. "A proposed moddification of the Germano subgridscale closure method", Phys.Fluids A4 (3), pp. 633-635.

Oshima, M., Kobayashi, T., Taniguchi, N., Tsubokura, M., 1996. "Development of Filtering Operation for Dynamic SGS Model Using Finite Element Method". The Second ERCOFTAC Workshop on Direct and Large Eddy Simulation. Septembre 16-19, 1996. Grenoble, Franca.

Padilla, E. L. M., Silveira Neto, A., 2000. "Numerical Implementation of the Dynamic Model for Turbulent Flows in Cylindrical Coordinates" (in Portuguese). IV Simpósio Mineiro de Mecânica Computacional. Uberlândia, MG, Brazil. pp. 573-580.

Petry, A.P., Awruch, A.M., 2003. "Numerical Analysis of TreeDimensional Turbulent Flow by the Finite Element Method and Large Eddy Simulation", In COBEM2003, $17^{\text {th }}$ International Congress of Mechanical Engineering, ABCM, SP, Brazil.

Petry, A.P., 2002. "Numerical Analysis of Tree-Dimensional Turbulent Flows Using the Finite Element Method and Large Eddy Simulation"(in Portuguese), DSc. Thesis. Programa de Pós-Graduacão em Engenharia Mecânica, Universidade Federal do Rio Grande do Sul, Porto Alegre, RS Brazil, 135 pages.

Petry, A.P., Awruch, A.M., 1997. "Turbulent Flows Simulation Using the Finite Element Method and Large Eddy Simulation"(in Portuguese), ENIEF 97, Proceedings, San Carlos de Bariloche, Argentina.

Prasad. A. K., Koseff, J. R., 1989. "Reynolds Number and End-wall Effects on a Lid-driven Cavity Flow". Phys. Fluids A, Vol.1, No. 2, pp 208-218.

Reddy, J.N., Gartling, D.K.. 1994. The Finite Element Method in Heat Transfer and Fluid Dynamics, CRC Press.

Rogallo, R.S.; Moin, P., 1984. Numerical Simulation of Turbulent Flows, Ann. Rev. Fluid Mechanics, Vol.16 pp. 99-137.

Schlichting, H. , 1968. "Boundary Layer Theory", McGraw-Hill, New York, 1968.

Silveira Neto, A. 2002, "Fluid Turbulence Fundamentals", Turbulence: Proceeding of the First Spring School in Transition and Turbulence, Editors: Freire, A.P.S., Menut P.P.P e Su, J., ABCM, Rio de Janeiro, Brazil.

Silveira Neto, A. Grand, D., Métais, O., Lesieur,M., 1993 “A Numerical Investigation of Coherent Vortices in Turbulence Behind a Backward-Facing Step", Journal of Fluid Mechanics, Vol.256, pp. 1-25.

Smagorinsky, J. 1963. "General Circulation Experiment with the Primitive Equations. I the Basic Experiment", Monthly Weathther Review, Vol.91,n. 3, pp 99-164. Press.

Tenekes, H.; Lumley,J.L., 1972. A First Course in Turbulence, MIT

White, F.M., 1974. "Viscous Fluid Flow", McGraw-Hill, New York.

Williams,P.T., Baker, A.J., 1997. "Numerical Simulations of Laminar Flow over a 3D Backward-Facing Step", International Journal for Numerical Methods in Fluids, Vol.24, pp. 1159-1183.

Zang, Y., Street, R. L., Koseff, J. R., 1993. “A Dynamic Mixed SubgridScale Model and its Application to Turbulent Recirculating Flows". Phys. Fluids A, Vol. 5, No. 12, pp. 3186-3196. 